\title{
The Microarcsecond Quasar J1819+3845
}

\author{
J. Dennett-Thorpe ${ }^{1}$ \& A. G. de Bruyn ${ }^{2,1}$ \\ (1) Kapteyn Institute, Postbus 800, Groningen, The Netherlands; (2) \\ ASTRON, Postbus 2, Dwingeloo, The Netherlands
}

\begin{abstract}
We present new WSRT observations of the micro-arcsecond quasar J1819+3845. All short term variations are attributed to interstellar scintillation of a source which is at most 30 micro-arcseconds in diameter. The timescale of the modulations changes over the year, which we interpret as due to a peculiar velocity of the scattering medium. The scintillation behaviour can be used to determine sub-structure in the source.
\end{abstract}

\section{Introduction}

The quasar $\mathrm{J} 1819+3845$ is, at radio wavelengths, the most extremely variable extragalactic source known (Dennett-Thorpe \& de Bruyn, 2000 ApJL 529 65). At the beginning of 1999 it showed peak to peak variations of more than $600 \%$ at $5 \mathrm{GHz}$, with just an hour between the minima. Intrinsic variations of this rapidity would require not only absurd brightness temperatures, but a source which would be small enough to scintillate due to the Galactic interstellar medium. We therefore consider refractive scintillations as the only cause of the hourly variations and concluded that:

- the scattering occurs in a nearby $(\sim 25 \mathrm{pc})$ screen

- $>55 \%$ of the flux density at $5 \mathrm{GHz}$ must be contained within a diameter of 30 microarcseconds: or 5 light-months at the source redshift $(z=0.53)$.

- If the scintillations are due to one component, the brightness temperature $\mathrm{T}_{B}=510^{12} \mathrm{~K}$

This high $\mathrm{T}_{B}$ combined with a spectral peak $\sim 100 \mathrm{GHz}$, requires that the source is a transient phenomenon ( $<5$ months); has an exotic emission process; or has Doppler factors of $\sim 15$ and a continuous energy input.

We conducted a monitoring campaign with the WSRT to address the velocity of the scattering plasma (a critical unknown in the application of the scintillation theory), the longevity of the source and the source structure.

\section{Seasonal variations}

From the monitoring campaign we found that:

- The source continued to scintillate throughout the 14 month observing period.

- There is a yearly variation in the timescale of the modulations over this period; with the fastest variations around February, and the slowest in August. The variations in August are around an order of magnitude slower than those in 
February.

- There is no indication for a change in the strength of the modulations.

These results most easily explained as due to a seasonal change in the relative velocity of the Earth and the scattering plasma. This changes the speed at which we move through the projected scintillation pattern. As J1819+3845 is very close to the ecliptic pole, there is very little change in the timescale of the modulations due to the Earth's motion. We conclude, from fits to the observed timescale (measured in several different manners), that the scattering plasma has a velocity $\sim 25 \mathrm{~km} \mathrm{~s}^{-1}$ w.r.t. the LSR. This velocity is in the opposite sense expected due to differential Galactic rotation, and further strengthens the notion that the scattering occurs predominantly in a discrete structure, not throughout the ISM.

The scintillations from a finite source are weighted towards the medium where the refractive scale (medium dependent), Fresnel size (distance dependent) and source size are matched. Thus quasars (unlike pulsars) will be more affected by nearby scattering material.

We note that the character of the modulations appears different around May (smaller, faster variations accompanied with larger excursions) than at other times of the year (where the light curves seem smoother). Furthermore, the period of slow modulations, which extends for almost 6 months, is longer than expected if the change in timescale is solely due to the peculair velocity of the plasma. Work is underway to investigate that, in addition to the peculiar velocity of the plasma, there may be additional affects due to source elongation, anisotropic turbulence or contributions from other scattering media (including the extended Galactic ISM).

\section{Implications for the AGN}

-We have now measured the effective velocity of the scattering medium, and have therefore eliminated a previous unknown in our calculation. Assuming that all the flux density in the 'scintillating component' $(75 \mathrm{mJy}$ at $5 \mathrm{GHz}$ ) is in a single component, we have a firm limit $\mathrm{T}_{B}>510^{12} \mathrm{~K}$.

- There is evidence for source structure. As the modulations do not reach $100 \%$ at any frequency, the source must have a component which is larger than the scattering zone, or $>30 \mu \operatorname{arcsecs}$ at $5 \mathrm{GHz}$.

Furthermore, there is evidence for substructure in the source, on scales $\sim$ the scattering zone, from the persistent asymmetries in the lightcurves. Variations in polarised flux also support multiple scintillating components.

-Doppler factors $\mathcal{D} \sim 15$ would allow for the Compton losses which limit the intrinsic $\mathrm{T}_{B}$. (If $\mathcal{D}$ does not greatly exceed this, the source should be Compton limited and therefore a copious X-ray emitter.) Even so, the spectral peak $100 \mathrm{GHz}$ means that these electrons would lose their energy in a matter of weeks.

-The source shows intrinsic variations in polarisation over a period of months, but at centimeter wavelengths, the intrinsic luminosity of the source has been remarkably constant. At most it has shown a $20 \%$ increase.

How the source remains so small, so hot, and also so constant is a puzzle. 